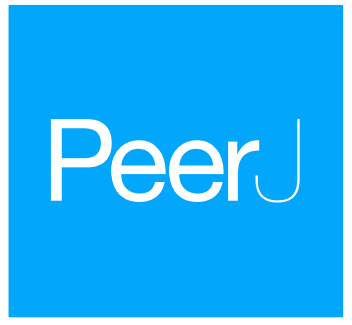

\title{
Biomechanical implications of skeletal muscle hypertrophy and atrophy: a musculoskeletal model
}

\author{
Andrew D. Vigotsky ${ }^{1,2}$, Bret Contreras ${ }^{3}$ and Chris Beardsley ${ }^{4}$ \\ ${ }^{1}$ Kinesiology Program, Arizona State University, Phoenix, AZ, United States of America \\ ${ }^{2}$ Leon Root, M.D. Motion Analysis Laboratory, Hospital for Special Surgery, New York, NY, \\ United States of America \\ ${ }^{3}$ Sports Performance Research Institute New Zealand, Auckland University of Technology, \\ Auckland, New Zealand \\ ${ }^{4}$ Strength and Conditioning Research Limited, London, United Kingdom
}

\section{ABSTRACT}

Muscle hypertrophy and atrophy occur frequently as a result of mechanical loading or unloading, with implications for clinical, general, and athletic populations. The effects of muscle hypertrophy and atrophy on force production and joint moments have been previously described. However, there is a paucity of research showing how hypertrophy and atrophy may affect moment arm (MA) lengths. The purpose of this model was to describe the mathematical relationship between the anatomical cross-sectional area (ACSA) of a muscle and its MA length. In the model, the ACSAs of the biceps brachii and brachialis were altered to hypertrophy up to twice their original size and to atrophy to one-half of their original size. The change in MA length was found to be proportional to the arcsine of the square root of the change in ACSA. This change in MA length may be a small but important contributor to strength, especially in sports that require large joint moments at slow joint angular velocities, such as powerlifting. The paradoxical implications of the increase in MA are discussed, as physiological factors influencing muscle contraction velocity appear to favor a smaller MA length for high velocity movements but a larger muscle MA length for low velocity, high force movements.

Submitted 11 August 2015 Accepted 11 November 2015 Published 30 November 2015

Corresponding author

Andrew D. Vigotsky, avigotsky@gmail.com

Academic editor

John Hutchinson

Additional Information and Declarations can be found on page 12

DOI 10.7717/peerj.1462

Copyright

2015 Vigotsky et al.

Distributed under

Creative Commons CC-BY 4.0

\section{OPEN ACCESS}

Subjects Bioengineering, Computational Biology, Anatomy and Physiology, Kinesiology, Orthopedics

Keywords Moment arm, strength, Normalized force, Pennation angle, Muscle architecture, Biceps brachii, Brachialis, Elbow flexors, Torque, Moment

\section{INTRODUCTION}

Muscle hypertrophy is a common adaptation to mechanical loading, typically delivered in the form of long-term programs of resistance training. Muscle atrophy is a response to disuse that occurs quickly following even short periods of mechanical unloading, which can be as little as one week of strict bed rest (Dirks et al., 2015). Both muscle hypertrophy and atrophy have important implications for clinical, general, elderly and athletic populations, because of the relationship between measures of muscle mass or size and a range of performance and health outcomes. For example, among strength athletes, measures of muscle mass or size have been found to be very good predictors 
of Olympic weightlifting, powerlifting, and strongman performance (Brechue \& Abe, 2002; Siahkouhian \& Hedayatneja, 2010; Winwood, Keogh \& Harris, 2012). In the elderly, Janssen et al. (2004) found that low levels of muscle mass were strongly correlated with an increased risk of disability, Malkov et al. (2015) reported that reducing thigh muscle size was associated with an increased risk of hip fracture, and Srikanthan \& Karlamangla (2014) found that low levels of muscle mass were associated with increased all-cause mortality. In various clinical populations, reports have been made of increasing mortality or re-hospitalization rates in individuals with lower levels of muscle mass (Greening et al., 2014; Streja et al., 2011; Weijs et al., 2014).

The relationships between muscle size, or more accurately muscle cross-sectional area (CSA), and measures of performance or disability are underpinned by the unique ability of muscle to produce force, with greater muscle CSA corresponding to greater force production. At the individual muscle level, force is produced by the contractions of single muscle fibers, which are collected together in groups, known as fascicles. Muscle CSA is measured in two ways: anatomical CSA (ACSA) and physiological CSA (PCSA). ACSA is the muscle CSA measured in the plane perpendicular to its tendons (the longitudinal axis), commonly recorded at the widest point along the muscle. PCSA is the muscle CSA measured perpendicular to the muscle fascicles, which can vary for different parts of a pennate muscle. The angle between the longitudinal axis and the direction of the muscle fascicles is the fascicle pennation angle $\left(\theta_{p}\right)$. The component of force exerted by the muscle fascicles is $\cos \theta_{p}$, and therefore, as $\theta_{p}$ increases, this leads to a progressively smaller component of muscle force being transmitted along the longitudinal axis to the tendons. Consequently, where changes in muscle PCSA occur following a period of either mechanical loading or unloading, the effect on muscle force transmitted to the tendons will also be affected by any simultaneous changes in $\theta_{p}$, with the increases that occur following mechanical loading reducing the beneficial effect of hypertrophy, and the decreases that occur following unloading mitigating some of the adverse effects of atrophy (Aagaard et al., 2001; Erskine, Fletcher \& Folland, 2014; Kawakami et al., 1995; Seynnes, De Boer \& Narici, 2006). In order to accurately predict joint moments, it is important for musculoskeletal models to take into account all of the aforementioned architectural variables.

Such changes in muscle PCSA, ACSA, $\theta_{p}$, and fascicle length that follow from exposure to mechanical loading have been well documented (Aagaard et al., 2001; Erskine, Fletcher \& Folland, 2014; Kawakami et al., 1995; Seynnes, De Boer \& Narici, 2006). The changes that simultaneously occur in various measures of strength are often attributed to these architectural and morphological changes, in addition to neural adaptations (Behm, 1995). Yet, the change in muscle size appears to be one of the most important, if not the most important factor: Erskine, Fletcher \& Folland (2014) reported that changes in both muscle volume and muscle ACSA were strongly associated with increases in maximum voluntary isometric contraction (MVIC) force following a 12-week period of elbow flexor resistance training. Although the MVIC force values reported by Erskine, Fletcher \& Folland (2014) were recorded using a linear force transducer, they are actually indicative of a net joint moment. Nevertheless, it is important to note that measures of MVIC force recorded 
externally using load cells (as in Erskine, Fletcher \& Folland (2014)) are not identical to the isometric muscle forces produced internally, as the external force reflects a net joint moment. Internal isometric muscle force is a product of the single muscle fiber force (also called specific tension), PCSA and $\theta_{p}$. The internal isometric muscle force is transmitted along the longitudinal axis of the muscle through the tendons to act on the bones at either side of a joint, where it creates an isometric moment about the joint. This isometric joint moment is the product of the internal isometric muscle force and the moment arm (MA) length. The MA length is the perpendicular distance between the muscle line of action to the joint center of rotation. Consequently, changes in both internal isometric muscle force and MA length can affect the magnitude of the maximum isometric joint moment that can be produced.

Although muscle ACSA appears to be a key determinant of MVIC moment production, the changes in MA length that occur as a result of either hypertrophy or atrophy have been less well described. Nevertheless, it appears that there is a relationship between agonist muscle size and the MA associated with the joint action. Sugisaki et al. (2010) and Akagi et al. (2012) described a positive correlation between muscle size and muscle MA length. These findings suggest that larger muscles are likely to benefit from a longer MA and consequently that hypertrophy may lead to increases in MA length and atrophy to decreases in MA length, which may have impacts on the joint moment that go above and beyond alterations in muscle force. Indeed, Sugisaki et al. (2015) noted a small increase in triceps brachii MA length following hypertrophy of around 5.5\%, albeit which they deemed to be small and possibly negligible.

Since the relationship between MA length and muscle size is not fully understood, the purpose of this paper is to develop a two-dimensional mathematical model to describe how changes in ACSA of the proximal elbow flexors change MA length, $\theta_{p}$, and joint moment contributions.

\section{MATERIALS AND METHODS}

A mathematical model that related the ACSA of both BIC and BRA to elbow flexion joint moment contributions was created using WolframAlpha (Wolfram Research, Champaign, IL, USA) and Excel (Microsoft, Seattle, USA), and the regression was tested in Excel and Stata (StataCorp, College Station, TX, USA).

\section{Development of the musculotendinous unit}

A position-elbow flexor ACSA hyperbolic cosine regression equation was extrapolated from West et al. (2010), wherein magnetic resonance images (MRIs) were taken of the elbow flexors of 65 subjects, from 2.4 to $11.2 \mathrm{~cm}$ proximal to the elbow, in both elbow extension and in a neutral radioulnar joint position.

Let $x$ be distance from the elbow, from distal to proximal, in centimeters $(\mathrm{cm})$. Let

$\operatorname{ACSA}(x)=-\cosh (0.48(x-7.3))+23$

Therefore, the radius, assumed to be the centroid and average line of pull of the proximal elbow flexors, assumed to be a cylinder (Van der Linden et al., 1998), can be defined as a 


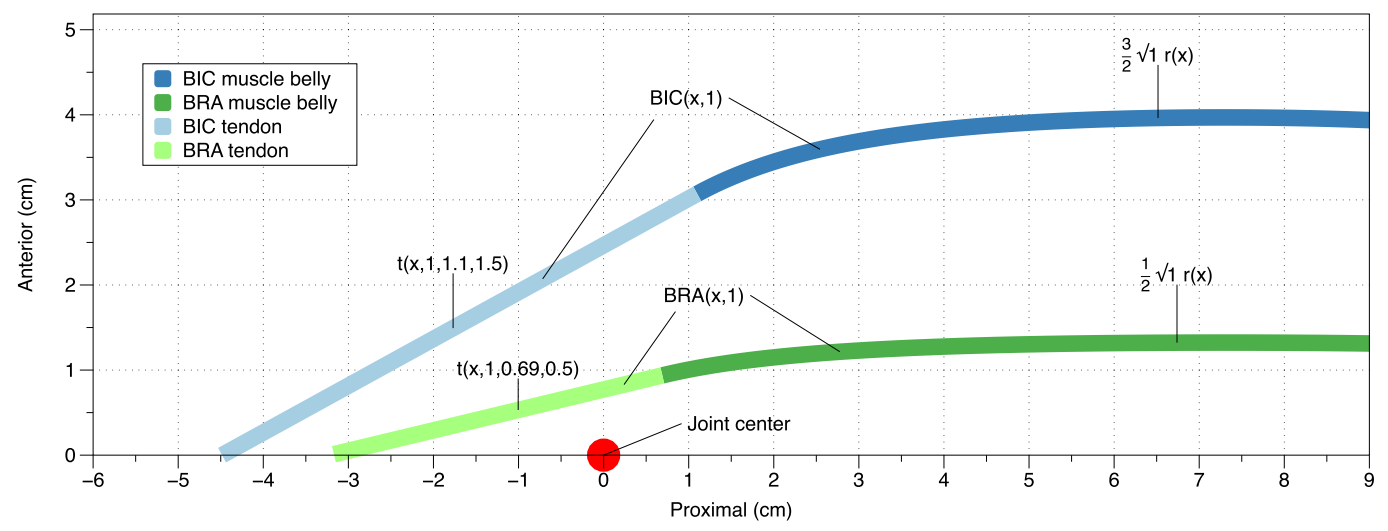

Figure 1 Graphical visualization of the model. $x$ represents the proximal distance from the elbow joint. The muscle bellies end at the point where the tangent line, $t$, representative of the tendon, would insert into the appropriate location distal to the elbow. Moment arms were calculated as the perpendicular distance from the joint center (origin) to the tendon $t$. Parameters for $t$ represent the position proximal to the elbow, coefficient of hypertrophy, $x_{\max }$, and anterior/superficial position. Parameters for BRA and BIC represent the position proximal to the elbow and coefficient of hypertrophy. The parameter of $r$ simply represents the position proximal to the elbow.

function of $\operatorname{ACSA}(x)$ :

$r(x)=\sqrt{\frac{\operatorname{ACSA}(x)}{\pi}}$.

Let $\alpha=$ coefficient of hypertrophy, or the degree to which the muscle is hypertrophying (or atrophying) from baseline, which assumes uniform growth across the muscle belly.

Let $\beta=$ the $x$ position of the musculotendinous (MT) junction. The centroids of the distal BIC and BRA tendons, tangent to the centroid of the distal-most section of each muscle belly $(t)$, assumed to be the line of pull, can be described as

$t(x, \alpha, \beta, \varphi)=\frac{d r}{d x}(\beta) x+\varphi \sqrt{\alpha} r(\beta)-\beta \frac{d r}{d x}(\beta)$

where $\varphi$ is a constant to correct for muscle location. More specifically, it was assumed that the BIC lay directly over (superficial and anterior to) the BRA. Because Eq. (1) represents the ACSA of both the BIC and BRA, the centroid of this (Eq. (2)) represents the division between the BIC and BRA, under the assumption that the muscles are equal in size. Therefore, when $\varphi=0.5$, that represents half of the position of the centroid derived from Eq. (1), and when $\varphi=1.5$, that represents one and a half times the position of the centroid derived from Eq. (1). Under the aforementioned assumptions, the former would represent the BRA, and the latter represents the BIC. The Cartesian outcomes of $t$ and $r$ in context of the model are illustrated in Fig. 1. The mathematical definitions of BIC and BRA depicted in Fig. 1 are described in the proceeding paragraphs.

Because $r(x)$ represents the centroid of both the BIC and BRA, it is necessary to divide this into each individual muscle. The BIC was set to begin $1.1 \mathrm{~cm}$ proximal to the joint center in order to control for insertion point, which was fixed $4.51 \mathrm{~cm}$ distal to the axis of 
rotation (capitulum). This was assumed to be approximately the center of the insertion site, as the capitulum has a $1.06 \mathrm{~cm}$ radius (Shiba et al., 1987), the bicipital tuberosity is $2.5 \mathrm{~cm}$ distal from the radial head, and the insertion site is $2.2 \mathrm{~cm}$ long (Mazzocca et al., 2006). As per the MRIs, the muscle belly was set to end $11.2 \mathrm{~cm}$ proximal to the elbow joint. Therefore, the BIC MT unit (MTU) can be described as

$\operatorname{BIC}(x, \alpha)= \begin{cases}t\left(x, \alpha, 1.1, \frac{3}{2}\right) & x<1.1 \\ \frac{3}{2} \sqrt{\alpha} r(x) & 1.1 \leq x \leq 11.2 .\end{cases}$

The muscle belly of the BRA was set to begin $0.69 \mathrm{~cm}$ proximal to the joint center in order to control for insertion point, which was fixed $3.17 \mathrm{~cm}$ distal to the axis of rotation (trochlea). Like the BIC, it was assumed that this was the center of the insertion site, as the trochlea has a $0.75 \mathrm{~cm}$ radius (Murray, Buchanan \& Delp, 2002), the coronoid process is about $1.10 \mathrm{~cm}$ from the trochlea, and the insertion site is about $2.63 \mathrm{~cm}$ long (Cage et al., 1995). Therefore, the MTU of the BRA can be described as

$\operatorname{BRA}(x, \alpha)= \begin{cases}t\left(x, \alpha, 0.69, \frac{1}{2}\right) & x<0.69 \\ \frac{1}{2} \sqrt{\alpha} r(x) & 0.69 \leq x \leq 11.2 .\end{cases}$

Both the BIC and BRA were modeled on the interval $0.5 \leq \alpha \leq 2.0$, with a step of 0.1 .

\section{Moment arm, force, pennation angle, and normalized muscle force calculations}

The joint center of the elbow is represented by the origin $(0,0)$, and the perpendicular distance from the tendon to the joint center was then calculated as the MA. This was done so by finding the angle of insertion via arctangent and using that angle to find the vertical, or perpendicular, component by multiplying the lever arm by the sine of the insertion angle (Eq. (6)).

$\mathrm{MA}=\gamma \cdot \sin \left(\arctan \left(\frac{\partial(\mathrm{BIC} \vee \mathrm{BRA})}{\partial x}(\beta, \alpha)\right)\right)$

where $\gamma$ is the muscle's insertion point.

Increases in pennation angle were assumed to occur proportionally with increases in maximum ACSA (Erskine, Fletcher \& Folland, 2014). In order to calculate muscle force, normalized muscle force (NMF) was assumed to be $30.75 \mathrm{~N} \mathrm{~cm}^{-2}$, which is the average of previously reported values $\left(23,30,33\right.$, and $37 \mathrm{~N} \mathrm{~cm}^{-2}$ ) (Edgerton, Apor \& Roy, 1990; Ikai \& Fukunaga, 1968; Nygaard et al., 1983; Ralston et al., 1949), and was assumed to remain constant with changes in ACSA. Muscle force ( $\left.F_{\text {muscle }}\right)$ was derived from NMF by multiplying NMF by the ACSA (Eq. (7)). The use of ACSA rather than PCSA is appropriate in this context, as Kawakami et al. (1994) found no statistical differences between ACSA 
Table 1 Anatomical cross-sectional areas, moment arm lengths, and moment contributions. Taking into account the changing moment arm broadens the elbow flexion moment contribution range of both the biceps brachii and brachialis.

\begin{tabular}{llllll} 
& Atrophy & $\boldsymbol{\Delta}_{\mathbf{A B}}$ & Baseline & $\boldsymbol{\Delta}_{\mathbf{H B}}$ & Hypertrophy \\
\hline BIC ACSA $\left(\mathrm{cm}^{2}\right)$ & 5.50 & -5.50 & 11.00 & +11.00 & 22.00 \\
BRA ACSA $\left(\mathrm{cm}^{2}\right)$ & 5.50 & -5.50 & 11.00 & +11.00 & 22.00 \\
BIC MA length $(\mathrm{cm})$ & 1.63 & -0.54 & 2.17 & +0.59 & 2.76 \\
BRA MA length $(\mathrm{cm})$ & 0.54 & -0.21 & 0.75 & +0.28 & 1.03 \\
BIC moment contribution (N m) (incl. MA) & 2.73 & -4.34 & 7.07 & +9.10 & 16.17 \\
BRA moment contribution (N m) (incl. MA) & 0.9 & -1.59 & 2.49 & +4.05 & 6.54 \\
BIC moment contribution (N m) (excl. MA) & 3.63 & -3.44 & 7.07 & +5.62 & 12.69 \\
BRA moment contribution (N m) (excl. MA) & 1.26 & -1.23 & 2.49 & +2.27 & 4.76 \\
\hline
\end{tabular}

and PCSA of the elbow flexors.

$F_{\text {muscle }}=\mathrm{ACSA} \cdot \mathrm{NMF}$.

Tendon force $\left(F_{\text {tendon }}\right)$ was then calculated as the parallel component of the muscle's force (Eq. (8)).

$F_{\text {tendon }}=F_{\text {muscle }} \cdot \cos \theta_{p}$

\section{Moment contributions}

The elbow flexion moment contributions of the BIC and BRA $(M)$ were calculated by simply multiplying each muscle's tendon force by its respective moment arm.

$M=F_{\text {tendon }} \cdot \mathrm{MA}$.

\section{Validation}

In order to validate the model, the primary outcomes - that is, MA length, pennation angle, and joint moment trends_-were compared with previous, relevant literature.

\section{RESULTS}

Equation (1) showed a strong correlation with the length-ACSA relationship described by West et al. (2010) $(p<0.001 ; r=0.911)$. The results of the model, including the effects of ACSA on MA, elbow flexion moment contributions, pennation angle, muscle force, and tendon force, can be found in Fig. 2. The key data for the atrophy, baseline, and hypertrophy conditions are shown in Table 1.

BIC and BRA MA lengths increased approximately hyperbolically with increases in ACSA (Fig. 2A). Angle of pennation for both BIC and BRA increased linearly with increases in ACSA (Fig. 2B). Both the BIC and BRA were shown to have identical muscle forces (Fig. 2C), but due to the differences in pennation angles (Fig. 2B), the differences in tendon force became more apparent with greater muscle ACSA (Fig. 2D). Figure 2E depicts how changes in MA length affect elbow flexion moment contributions, as the dashed 

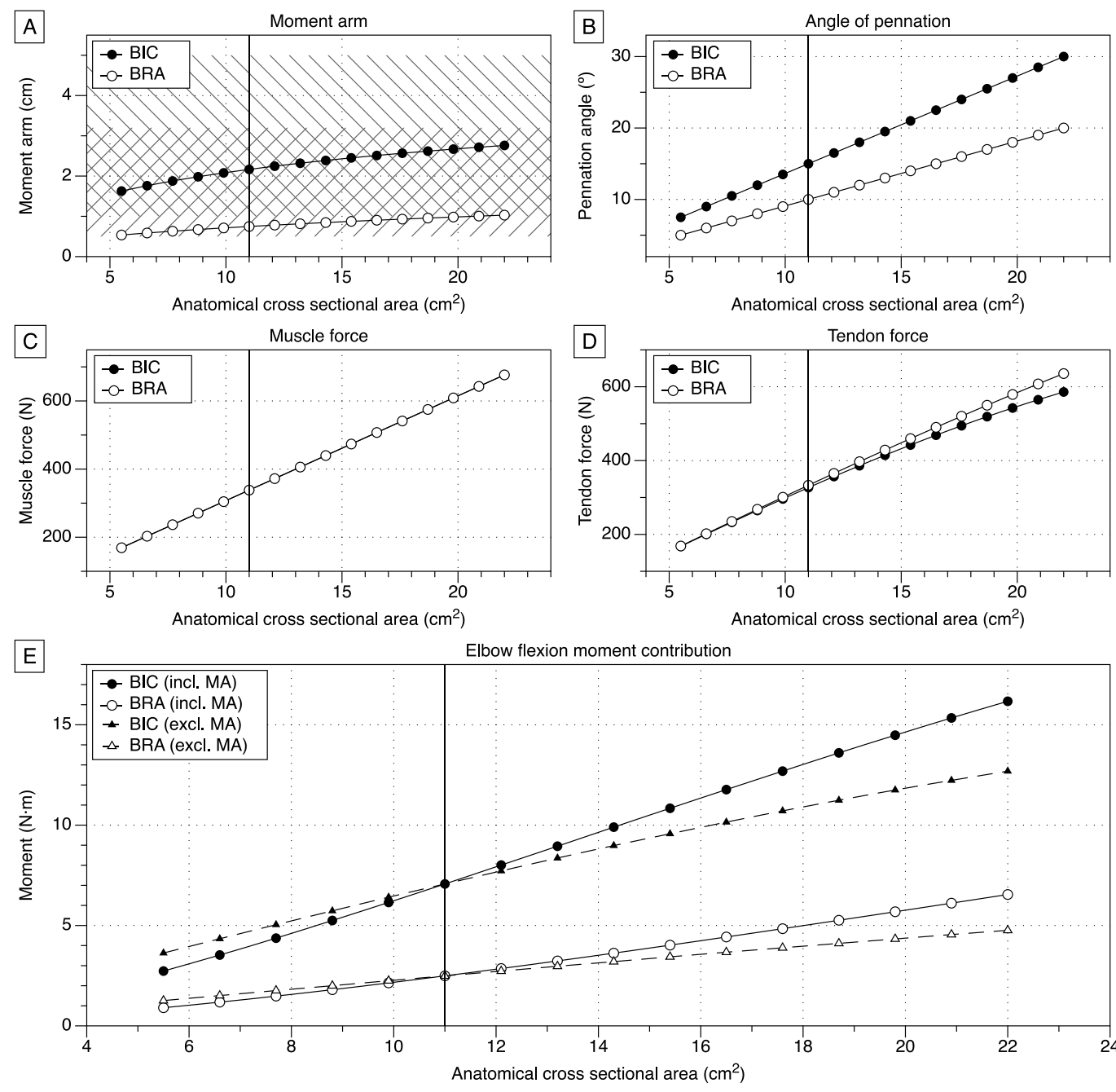

Figure 2 Biomechanical variables as a function of anatomical cross-sectional area. (A) Relationship between biceps brachii anatomical cross-sectional area and muscle moment arm. Negatively sloped lines are normal BIC MAs, and positively sloped lines are empirical BRA MAs (Ramsay, Hunter \& Gonzalez, 2009). (B) Pennation angles of the BIC and BRA increase linearly with increases in ACSA. (C) Muscle force increases with ACSA. (BIC plots are underneath, and identical to, the BRA plots.) (D) Tendon force increases with ACSA. (E) The elbow flexion moment contributions of the BIC and BRA with changes in ACSA, both with and without the changes in MA length. Vertical lines at $11.0 \mathrm{~cm}^{2}$ indicate baseline.

lines illustrate the moment contributions if the MA length did not change (remained identical to baseline $\left(\mathrm{ACSA}=11.0 \mathrm{~cm}^{2}\right)$ ). At $22.0 \mathrm{~cm}^{2}$, accounting for changes in BIC and BRA MA length result in $27.2 \%$ and $37.3 \%$ greater joint moment contributions, respectively (Fig. 2E).

\section{DISCUSSION}

The MA lengths of the BIC and BRA at baseline in this model were within previously reported ranges in some studies (An et al., 1981; Ramsay, Hunter \& Gonzalez, 2009), and although they may appear shorter than those reported by some other investigators (Amis, Dowson \& Wright, 1979; An et al., 1981; Edgerton, Apor \& Roy, 1990; Pauwels, 1980), most 
studies did not report MA lengths in the same joint positions utilized for this model. Furthermore, the MA lengths reported in this model are similar to those previously modeled by Murray, Delp \& Buchanan (1995) (Fig. 2A). The increases in pennation angles reflect values and trends previously reported, in that pennation angle increases linearly with increases in ACSA (Erskine, Fletcher \& Folland, 2014; Ikegawa et al., 2007; Kawakami, Abe \& Fukunaga, 1993; Kawakami et al., 1995) (Fig. 2B). Training studies corroborate the described increase in elbow flexion moment contributions, as it has been shown that net elbow flexion moments increase linearly with increases in ACSA (Erskine et al., 2010a; Erskine et al., 2010b).

To the authors' knowledge, this is the first model to describe the effects of muscle hypertrophy and atrophy on MA length. It revealed important changes in MA lengths of the BIC and BRA with increases in ACSA (Figs. 2A and 2E). Previous research has only attributed increases in net joint moment production to the effects of hypertrophy on muscle force (Aagaard et al., 2001; Erskine, Fletcher \& Folland, 2014), while ignoring potential changes in MA length, as described by our model. Intuitively, this change in MA length must be a function of a change in insertion angle, as the insertion point cannot shift. This increase in insertion angle occurs as the size of the muscle belly increases, thereby shifting the resultant vector of the muscle farther from the humerus and consequently from the joint center. This is exemplified by the ACSA at baseline $\left(11.0 \mathrm{~cm}^{2}\right)$ corresponding to MA lengths of $0.75 \mathrm{~cm}$ and $2.17 \mathrm{~cm}$ for the BRA and BIC, respectively, while the ACSA of a muscle double that size $\left(22.0 \mathrm{~cm}^{2}\right)$ corresponds to MA lengths of $1.03 \mathrm{~cm}$ and $2.76 \mathrm{~cm}$ for the BRA and BIC, respectively (Table 1, Fig. 3). In other words, for a $100 \%$ increase in ACSA, the MA lengths of the BIC and BRA increase by $27.2 \%$ and $37.3 \%$, respectively.

The modeled change in MA length for the BIC and BRA in this particular joint position (i.e., neutral radioulnar joint in elbow extension) is proportional to the arcsine of the square root of the change in ACSA $(\triangle \mathrm{MA} \propto \arcsin (\sqrt{\Delta \mathrm{ACSA}}))$. This is due to lengths being proportional to the square root of the area in which they are contained (as in Eq. (2)), while the arcsine controls for the perpendicular component (as in Eq. (6)). The relationship observed by Sugisaki et al. (2015) elucidates just how subtle changes in MA length can be, as a $33.6 \%$ increase in triceps brachii ACSA was accompanied by a $5.5 \%$ increase in MA length. It is unclear if other muscles, joints, or joint angles would behave similarly under hypertrophy, as these results cannot be extrapolated.

Although the increase in MA length appears to be beneficial for static or quasi-static strength, it may be detrimental to high velocity, dynamic movements. This is paradoxical, because a larger MA length should be helpful in producing a larger net joint moment, which might be expected to increase angular acceleration, irrespective of joint angular velocity. However, owing to the biomechanical properties of muscle, this is not the case. Specifically, in accordance with the hyperbolic force-velocity relationship, less force can be developed during a high velocity, dynamic muscle action than during a slow velocity or isometric muscle action (Hill, 1938). It is thought that the force-velocity relationship may arise as a result of a number of factors, including the number of cross-bridges (Piazzesi et al., 2007), fluid friction or viscosity (Gasser \& Hill, 1924; Hill, 1922), adenosine 


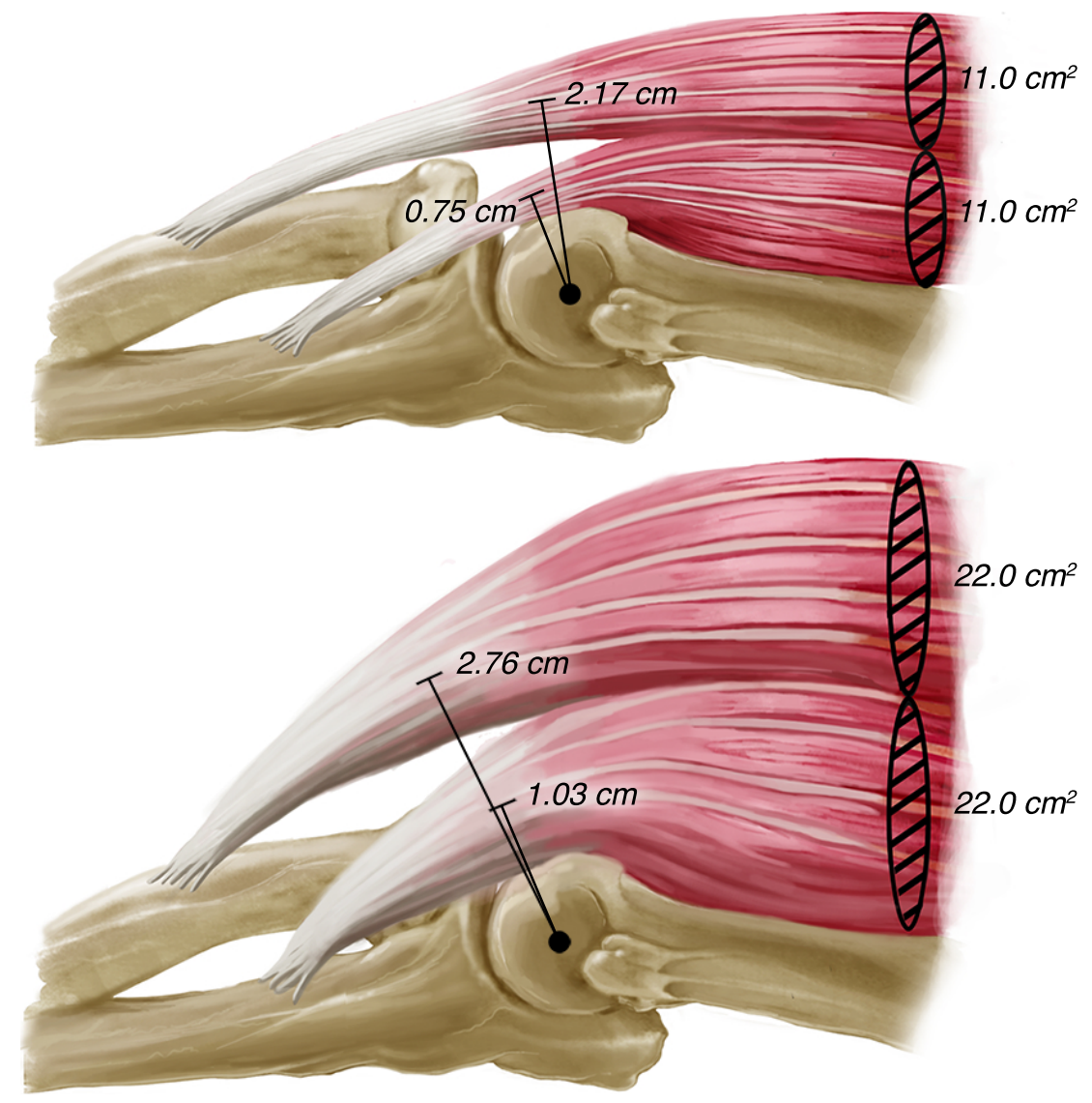

Figure 3 Illustration of the changes in biceps brachii and brachialis moment arm lengths with increases in anatomical cross-sectional area. By doubling the anatomical cross-sectional area of the biceps brachii and brachialis, the moment arms of each increase by $27.2 \%$ and $37.3 \%$, respectively.

diphosphate (ADP) dissociation rates (Nyitrai et al., 2006), and passive elastic tension at long lengths (Abbott \& Wilkie, 1953). Irrespective of the underlying mechanisms, however, Nagano \& Komura (2003) showed that a shorter MA length is beneficial for high velocity, dynamic muscle actions, since a given length change in the muscle will necessarily cause greater joint excursion. Consequently, with a shorter MA length, more muscle force can be produced, as a shorter change in length is required, which necessitates a smaller contraction velocity. Mathematically, this can be described by the equation for MA length, where $d l$ is the instantaneous change in MTU length and $d \theta$ is the instantaneous change in joint angular displacement (in radians) (Eq. (8)). With a larger MA length, a greater change in MTU length must occur for the same change in joint angular displacement, which would require a greater contraction velocity. The interactions of the ramifications of an increased MA on angular velocity and angular acceleration require further investigation, and may have several, important sport- or task-specific implications.

$\mathrm{MA}=\frac{d l}{d \theta}$ 


\section{LIMITATIONS}

There were a number of limitations inherent in this study that arose from the assumptions that were made. It was assumed that there was no change in either muscle fascicle or tendon length during the production of the calculated contributory elbow flexion joint moments, but changes in both muscle fascicle length and tendon elongation have been observed in vivo, in the tibialis anterior (Ito et al., 1998). Similarly, it was assumed that no myofascial force transfer occurred between the elbow flexors, although it has been observed that there are several ways in which this could occur (Huijing \& Jaspers, 2005).

Our model assumed no changes in NMF, which directly affects muscle force calculations and thus joint moment contributions. Although force production typically increases to a greater extent than ACSA, some studies have reported reductions in normalized force following resistance training, albeit not in the elbow flexors (Ikegawa et al., 2007; Kawakami et al., 1995; Sale, Martin \& Moroz, 1992). When considering studies performed in the elbow flexors, it is, however, apparent that normalized force typically either increases (Brandenburg \& Docherty, 2002; Vikne et al., 2006) or remains constant (Narici \& Kayser, 1995; Takarada et al., 2000). These equivocal findings indicate that our model may or may not reflect the typical changes in normalized force expected during resistance training for the elbow flexors, which may therefore arise due to central factors influencing strength gains or peripheral changes affecting single fiber specific tension.

Indeed, although excluded in our assumptions, studies have shown increases in single fiber specific tension using both in vivo (Erskine et al., 2011; Erskine et al., 2010a; Erskine et al., 2010b) and in vitro designs (Erskine et al., 2011; Pansarasa et al., 2009; Parente et al., 2008) following resistance training protocols, which were not necessarily accompanied by muscle hypertrophy. Changes in specific tension observed in vivo indicate that changes in either extracellular lateral force transmission or myofibrillar packing density might be responsible for alterations in normalized force, while changes observed in vitro can likely only be explained by alterations in myofibrillar packing density. Additionally, it has been reported that changes in specific tension occur in tandem with atrophy and that such changes appear to be associated with reduced myofibrillar packing density (Riley et al., 1998). Whether the exact same mechanisms are involved in the alteration of specific tension with mechanical loading and unloading, however, is unclear.

The use of a cylinder to model the BIC and BRA is not necessarily completely accurate, but cylindrical shapes have been used in previous muscle models, such as the gastrocnemius (Van der Linden et al., 1998). However, notwithstanding this point, the same proposed mathematical relationship would still stand with other, irregular shapes, as all linear distances within a cross-section are related to the square root of the area of that cross-section.

Our model assumed that there were no changes in fascicle length, changes in fiber type area proportion, or shifts in the fiber type of individual muscle fibers. However, previous research indicates that changes do occur in fascicle length (Baroni et al., 2013; Blazevich et al., 2003; Reeves et al., 2009) and in respect of proportional muscle fiber type areas (Campos et al., 2002; Schuenke et al., 2012; Staron et al., 1991), although the literature is currently 
equivocal regarding the exact nature of these responses to both resistance training and disuse atrophy (De Souza et al., 2014; Kawakami et al., 1995). In addition, the effect of muscle fiber type on maximal strength is likely less important than its effect on dynamic strength, as the specific tension of type I and type II muscle fibers is not normally found to be substantially different (e.g., Harber \& Trappe, 2008) but there is often a marked difference reported in muscle contraction velocity (e.g., Harber \& Trappe, 2008). However, it can be argued that the model is more robust and free from possible confounding factors because the aforementioned variables were not included, so they do not have to be "teased out."

Additionally, although excluded in our assumptions, changes in agonist voluntary activation or co-contraction might be expected to occur following periods of mechanical loading or unloading. Indeed, some studies have reported small increases in voluntary activation following resistance training (Ekblom, 2010; Erskine et al., 2010b), which may imply that mechanical loading involves changes in neural drive. Whether such increases are likely to have a substantial impact on strength gains given that voluntary activation levels of $>93 \%$ are frequently observed in young, untrained subjects before commencing resistance training (Erskine et al., 2010b; Power et al., 2015; Venturelli et al., 2015) appears questionable. Nevertheless, there is some evidence that voluntary activation is reduced in the elderly, possibly following sustained periods of disuse leading to atrophy (Klass, Baudry \& Duchateau, 2007), and there is good evidence that such reductions in voluntary activation can be reversed following sustained programs of resistance training (Arnold \& Bautmans, 2014). The effect of changes in antagonist co-contraction following periods of mechanical loading and unloading is much less clear. There does not appear to be any difference between antagonist co-contraction activity between trained and untrained individuals (Maeo et al., 2013), nor does antagonist co-contraction activity change following training in young (Maeo et al., 2014) or old (Arnold \& Bautmans, 2014) individuals. Like the changes in fascicle and fiber characters, it can also be argued that by not including these neural adaptations, the model includes less possible confounding variables. Therefore, the modeled changes in forces and moments are strictly due to the architectural characteristics included in the model.

Lastly, and perhaps most importantly, this model only examined two muscles in one joint position (i.e., neutral). It is likely that with changing joint positions, the relationship would shift (Murray, Delp \& Buchanan, 1995). For example, during elbow flexion, BIC and BRA MA lengths are greater because the insertion angle approaches $90^{\circ}$, so greater hypertrophy may be disadvantageous for MA length in such positions, as it may shift the insert angle away from $90^{\circ}$. However, such circumstances have yet to be described and modeled.

\section{CONCLUSIONS}

The contribution of changes in ACSA to joint moment contributions following hypertrophy and atrophy resulting from mechanical loading and unloading are not fully understood, nor are the implications of concomitant changes in MA length. This model was the first to describe how changes in ACSA following hypertrophy or atrophy of the BIC and BRA might alter MA length and how both changes in ACSA and MA length 
might impact on relative elbow flexion moment contributions in neutral radioulnar and elbow joint positions. The results of this model should be interpreted with caution, as the predicted outcomes (namely, MA lengths) have not been demonstrated in vivo. Additionally, only one joint position (neutral) was investigated on two muscles, so the results cannot be extrapolated to other muscles or joint positions. Nevertheless, this model may serve as an effective tool for generating hypotheses that may inform experimental research with biomechanical implications.

\section{ACKNOWLEDGEMENTS}

We would like to thank Professor Stuart Phillips, for providing the position-CSA data necessary to complete this model, and Dr. Silvia Blemker, for reviewing and critiquing an early rendition of our model.

\section{ADDITIONAL INFORMATION AND DECLARATIONS}

\section{Funding}

The authors received no funding for this work.

\section{Competing Interests}

The authors declare there are no competing interests. Chris Beardsley is an employee of Strength and Conditioning Research Limited.

\section{Author Contributions}

- Andrew D. Vigotsky conceived and designed the experiments, performed the experiments, analyzed the data, contributed reagents/materials/analysis tools, wrote the paper, prepared figures and/or tables, reviewed drafts of the paper.

- Bret Contreras conceived and designed the experiments, wrote the paper, reviewed drafts of the paper.

- Chris Beardsley wrote the paper, prepared figures and/or tables.

\section{Data Availability}

The following information was supplied regarding data availability:

We have included the dataset in Supplemental Information.

\section{Supplemental Information}

Supplemental information for this article can be found online at http://dx.doi.org/ 10.7717/peerj.1462\#supplemental-information.

\section{REFERENCES}

Aagaard P, Andersen JL, Dyhre-Poulsen P, Leffers AM, Wagner A, Magnusson SP, Halkjaer-Kristensen J, Simonsen EB. 2001. A mechanism for increased contractile strength of human pennate muscle in response to strength training: changes in muscle architecture. The Journal of Physiology 534:613-623 DOI 10.1111/j.1469-7793.2001.t01-1-00613.x. 
Abbott BC, Wilkie DR. 1953. The relation between velocity of shortening and the tension-length curve of skeletal muscle. Journal of Physiology 120:214-223

DOI 10.1113/jphysiol.1953.sp004886.

Akagi R, Iwanuma S, Hashizume S, Kanehisa H, Yanai T, Kawakami Y. 2012. In vivo measurements of moment arm lengths of three elbow flexors at rest and during isometric contractions. Journal of Applied Biomechanics 28(1):63-69.

Amis A, Dowson D, Wright V. 1979. Muscle strengths and musculoskeletal geometry of the upper limb. Engineering in Medicine 8:41-48 DOI 10.1243/EMED_JOUR_1979_008_010_02.

An KN, Hui FC, Morrey BF, Linscheid RL, Chao EY. 1981. Muscles across the elbow joint: A biomechanical analysis. Journal of Biomechanics 14:659-669

DOI 10.1016/0021-9290(81)90048-8.

Arnold P, Bautmans I. 2014. The influence of strength training on muscle activation in elderly persons: a systematic review and meta-analysis. Experimental Gerontology 58:58-68 DOI 10.1016/j.exger.2014.07.012.

Baroni BM, Geremia JM, Rodrigues R, De Azevedo Franke R, Karamanidis K, Vaz MA. 2013. Muscle architecture adaptations to knee extensor eccentric training: rectus femoris vs. vastus lateralis. Muscle and Nerve 48:498-506 DOI 10.1002/mus.23785.

Behm DG. 1995. Neuromuscular implications and applications of resistance training. Journal of Strength and Conditioning Research 9(4):264-274 DOI 10.1519/00124278-199511000-00014.

Blazevich AJ, Gill ND, Bronks R, Newton RU. 2003. Training-specific muscle architecture adaptation after 5-wk training in athletes. Medicine and Science in Sports and Exercise 35:2013-2022 DOI 10.1249/01.MSS.0000099092.83611.20.

Brandenburg JP, Docherty D. 2002. The effects of accentuated eccentric loading on strength, muscle hypertrophy, and neural adaptations in trained individuals. Journal of Strength and Conditioning Research 16:25-32.

Brechue WF, Abe T. 2002. The role of FFM accumulation and skeletal muscle architecture in powerlifting performance. European Journal of Applied Physiology 86:327-336 DOI 10.1007/s00421-001-0543-7.

Cage DJN, Abrams RA, Callahan JJ, Botte MJ. 1995. Soft tissue attachments of the ulnar coronoid process: an anatomic study with radiographic correlation. Clinical Orthopaedics and Related Research 320:154-158.

Campos GE, Luecke TJ, Wendeln HK, Toma K, Hagerman FC, Murray TF, Ragg KE, Ratamess NA, Kraemer WJ, Staron RS. 2002. Muscular adaptations in response to three different resistance-training regimens: specificity of repetition maximum training zones. European Journal of Applied Physiology 88:50-60 DOI 10.1007/s00421-002-0681-6.

De Souza EO, Tricoli V, Aoki MS, Roschel H, Brum PC, Bacurau AV, Silva-Batista C, Wilson JM, Neves Jr M, Soares AG, Ugrinowitsch C. 2014. Effects of concurrent strength and endurance training on genes related to myostatin signaling pathway and muscle fiber responses. Journal of Strength and Conditioning Research 28:3215-3223 DOI 10.1519/JSC.0000000000000525.

Dirks M, Wall B, Goossens G, Van de Valk B, Van Loon L. 2015. One week of bed-rest substantially reduces muscle mass and induces insulin resistance in healthy, young males. The FASEB Journal 29:825-827.

Edgerton VR, Apor P, Roy RR. 1990. Specific tension of human elbow flexor muscles. Acta Physiologica Hungarica 75:205-216. 
Ekblom MM. 2010. Improvements in dynamic plantar flexor strength after resistance training are associated with increased voluntary activation and V-to-M ratio. Journal of Applied Physiology 109:19-26 DOI 10.1152/japplphysiol.01307.2009.

Erskine RM, Fletcher G, Folland JP. 2014. The contribution of muscle hypertrophy to strength changes following resistance training. European Journal of Applied Physiology 114:1239-1249 DOI 10.1007/s00421-014-2855-4.

Erskine RM, Jones DA, Maffulli N, Williams AG, Stewart CE, Degens H. 2011. What causes in vivo muscle specific tension to increase following resistance training? Experimental Physiology 96:145-155 DOI 10.1113/expphysiol.2010.053975.

Erskine RM, Jones DA, Williams AG, Stewart CE, Degens H. 2010a. Inter-individual variability in the adaptation of human muscle specific tension to progressive resistance training. European Journal of Applied Physiology 110:1117-1125 DOI 10.1007/s00421-010-1601-9.

Erskine RM, Jones DA, Williams AG, Stewart CE, Degens H. 2010b. Resistance training increases in vivo quadriceps femoris muscle specific tension in young men. Acta Physiologica 199:83-89 DOI 10.1111/j.1748-1716.2010.02085.x.

Gasser H, Hill A. 1924. The dynamics of muscular contraction. Proceedings of the Royal Society of London B: Biological Sciences 96:398-437 DOI 10.1098/rspb.1924.0035.

Greening N, Harvey-Dunstan T, Williams J, Morgan M, Singh S, Steiner M. 2014. Lower limb muscle mass using ultrasound predicts re-hospitalisation following admission for acute exacerbations of chronic respiratory disease [Abstract]. European Respiratory Journal 44:417.

Harber M, Trappe S. 2008. Single muscle fiber contractile properties of young competitive distance runners. Journal of Applied Physiology 105:629-636 DOI 10.1152/japplphysiol.00995.2007.

Hill A. 1922. The mechanism of muscular contraction. Physiological Reviews 2:310-341.

Hill AV. 1938. The heat of shortening and the dynamic constants of muscle. Proceedings of the Royal Society B: Biological Sciences 126(843):136-195 DOI 10.1098/rspb.1938.0050.

Huijing PA, Jaspers RT. 2005. Adaptation of muscle size and myofascial force transmission: a review and some new experimental results. Scandinavian Journal of Medicine and Science in Sports 15:349-380 DOI 10.1111/j.1600-0838.2005.00457.x.

Ikai M, Fukunaga T. 1968. Calculation of muscle strength per unit cross-sectional area of human muscle by means of ultrasonic measurement. Internationale Zeitschrift für Angewandte Physiologie Einschliesslich Arbeitsphysiologie 26:26-32.

Ikegawa S, Funato K, Tsunoda N, Kanehisa H, Fukunaga T, Kawakami Y. 2007. Muscle force per cross-sectional area is inversely related with pennation angle in strength trained athletes. Journal of Strength and Conditioning Research 22:128-131 DOI 10.1519/JSC.0b013e31815f2fd3.

Ito M, Kawakami Y, Ichinose Y, Fukashiro S, Fukunaga T. 1998. Nonisometric behavior of fascicles during isometric contractions of a human muscle. Journal of Applied Physiology 85:1230-1235.

Janssen I, Baumgartner RN, Ross R, Rosenberg IH, Roubenoff R. 2004. Skeletal muscle cutpoints associated with elevated physical disability risk in older men and women. American Journal of Epidemiology 159:413-421 DOI 10.1093/aje/kwh058.

Kawakami Y, Abe T, Fukunaga T. 1993. Muscle-fiber pennation angles are greater in hypertrophied than in normal muscles. Journal of Applied Physiology 74:2740-2744.

Kawakami Y, Abe T, Kuno SY, Fukunaga T. 1995. Training-induced changes in muscle architecture and specific tension. European Journal of Applied Physiology and Occupational Physiology 72:37-43 DOI 10.1007/BF00964112. 
Kawakami Y, Nakazawa K, Fujimoto T, Nozaki D, Miyashita M, Fukunaga T. 1994. Specific tension of elbow flexor and extensor muscles based on magnetic resonance imaging. European Journal of Applied Physiology and Occupational Physiology 68:139-147 DOI 10.1007/BF00244027.

Klass M, Baudry S, Duchateau J. 2007. Voluntary activation during maximal contraction with advancing age: a brief review. European Journal of Applied Physiology 100:543-551 DOI 10.1007/s00421-006-0205-X.

Maeo S, Takahashi T, Takai Y, Kanehisa H. 2013. Trainability of muscular activity level during maximal voluntary co-contraction: comparison between bodybuilders and nonathletes. PLoS ONE 8:e79486 DOI 10.1371/journal.pone.0079486.

Maeo S, Yoshitake Y, Takai Y, Fukunaga T, Kanehisa H. 2014. Effect of short-term maximal voluntary co-contraction training on neuromuscular function. International Journal of Sports Medicine 35:125-134 DOI 10.1055/s-0033-1349137.

Malkov S, Cawthon PM, Peters KW, Cauley JA, Murphy RA, Visser M, Wilson JP, Harris T, Satterfield S, Cummings S, Shepherd JA, Health ABCS. 2015. Hip fractures risk in older men and women associated with DXA-derived measures of thigh subcutaneous fat thickness, cross-sectional muscle area, and muscle density. Journal of Bone and Mineral Research 30:1414-1421 DOI 10.1002/jbmr.2469.

Mazzocca AD, Cohen M, Berkson E, Nicholson G, Carofino BC, Arciero R, Romeo AA. 2006. The anatomy of the bicipital tuberosity and distal biceps tendon. Journal of shoulder and elbow surgery/American Shoulder and Elbow Surgeons [et al] 16:122-127 DOI 10.1016/j.jse.2006.04.012.

Murray WM, Buchanan TS, Delp SL. 2002. Scaling of peak moment arms of elbow muscles with upper extremity bone dimensions. Journal of Biomechanics 35:19-26 DOI 10.1016/S0021-9290(01)00173-7.

Murray WM, Delp SL, Buchanan TS. 1995. Variation of muscle moment arms with elbow and forearm position. Journal of Biomechanics 28:513-525 DOI 10.1016/0021-9290(94)00114-J.

Nagano A, Komura T. 2003. Longer moment arm results in smaller joint moment development, power and work outputs in fast motions. Journal of Biomechanics 36:1675-1681 DOI 10.1016/S0021-9290(03)00171-4.

Narici MV, Kayser B. 1995. Hypertrophic response of human skeletal muscle to strength training in hypoxia and normoxia. European Journal of Applied Physiology and Occupational Physiology 70:213-219 DOI 10.1007/BF00238566.

Nygaard E, Houston M, Suzuki Y, Jorgensen K, Saltin B. 1983. Morphology of the brachial biceps muscle and elbow flexion in man. Acta Physiologica Scandinavica 117:287-292 DOI 10.1111/j.1748-1716.1983.tb07208.x.

Nyitrai M, Rossi R, Adamek N, Pellegrino MA, Bottinelli R, Geeves MA. 2006. What limits the velocity of fast-skeletal muscle contraction in mammals? Journal of Molecular Biology 355:432-442 DOI 10.1016/j.jmb.2005.10.063.

Pansarasa O, Rinaldi C, Parente V, Miotti D, Capodaglio P, Bottinelli R. 2009. Resistance training of long duration modulates force and unloaded shortening velocity of single muscle fibres of young women. Journal of Electromyography and Kinesiology 19:e290-e300 DOI 10.1016/j.jelekin.2008.07.007.

Parente V, D’Antona G, Adami R, Miotti D, Capodaglio P, De Vito G, Bottinelli R. 2008. Long-term resistance training improves force and unloaded shortening velocity of single muscle fibres of elderly women. European Journal of Applied Physiology 104:885-893 DOI 10.1007/s00421-008-0845-0. 
Pauwels F. 1980. Biomechanics of the locomotor apparatus: contribution on the functional anatomy of the locomotor apparatus. Berlin: Springer-Verlag.

Piazzesi G, Reconditi M, Linari M, Lucii L, Bianco P, Brunello E, Decostre V, Stewart A, Gore DB, Irving TC, Irving M, Lombardi V. 2007. Skeletal muscle performance determined by modulation of number of myosin motors rather than motor force or stroke size. Cell 131:784-795 DOI 10.1016/j.cell.2007.09.045.

Power GA, Makrakos DP, Stevens DE, Rice CL, Vandervoort AA. 2015. Velocity dependence of eccentric strength in young and old men: the need for speed! Applied Physiology, Nutrition, and Metabolism Physiologie Appliquée, Nutrition et Métabolisme 40:703-710 DOI 10.1139/apnm-2014-0543.

Ralston HJ, Polissar MJ, Inman VT, Close JR, Feinstein B. 1949. Dynamic features of human isolated voluntary muscle in isometric and free contractions. Journal of Applied Physiology 1(7): 526-533.

Ramsay JW, Hunter BV, Gonzalez RV. 2009. Muscle moment arm and normalized moment contributions as reference data for musculoskeletal elbow and wrist joint models. Journal of Biomechanics 42:463-473 DOI 10.1016/j.jbiomech.2008.11.035.

Reeves ND, Maganaris CN, Longo S, Narici MV. 2009. Differential adaptations to eccentric versus conventional resistance training in older humans. Experimental Physiology 94:825-833 DOI 10.1113/expphysiol.2009.046599.

Riley DA, Bain JL, Thompson JL, Fitts RH, Widrick JJ, Trappe SW, Trappe TA, Costill DL. 1998. Disproportionate loss of thin filaments in human soleus muscle after 17-day bed rest. Muscle and Nerve 21:1280-1289 DOI 10.1002/(SICI)1097-4598(199810)21:10<1280::AID-MUS6>3.0.CO;2-7.

Sale DG, Martin JE, Moroz DE. 1992. Hypertrophy without increased isometric strength after weight training. European Journal of Applied Physiology and Occupational Physiology 64:51-55 DOI 10.1007/BF00376440.

Schuenke MD, Herman JR, Gliders RM, Hagerman FC, Hikida RS, Rana SR, Ragg KE, Staron RS. 2012. Early-phase muscular adaptations in response to slow-speed versus traditional resistance-training regimens. European Journal of Applied Physiology 112:3585-3595 DOI 10.1007/s00421-012-2339-3.

Seynnes OR, De Boer M, Narici MV. 2006. Early skeletal muscle hypertrophy and architectural changes in response to high-intensity resistance training. Journal of Applied Physiology 102:368-373 DOI 10.1152/japplphysiol.00789.2006.

Shiba R, Sorbie C, Siu DW, Bryant JT, Cooke TD, Wevers HW. 1987. Geometry of the humeroulnar joint. Journal of Orthopaedic Research 6:897-906 DOI 10.1002/jor.1100060614.

Siahkouhian M, Hedayatneja M. 2010. Correlations of anthropometric and body composition variables with the performance of young elite weightlifters. Journal of Human Kinetics 25:125-131 DOI 10.2478/v10078-010-0040-3.

Srikanthan P, Karlamangla AS. 2014. Muscle mass index as a predictor of longevity in older adults. American Journal of Medicine 127:547-553 DOI 10.1016/j.amjmed.2014.02.007.

Staron RS, Leonardi MJ, Karapondo DL, Malicky ES, Falkel JE, Hagerman FC, Hikida RS. 1991. Strength and skeletal muscle adaptations in heavy-resistance-trained women after detraining and retraining. Journal of Applied Physiology 70:631-640.

Streja E, Molnar MZ, Kovesdy CP, Bunnapradist S, Jing J, Nissenson AR, Mucsi I, Danovitch GM, Kalantar-Zadeh K. 2011. Associations of pretransplant weight and muscle mass with mortality in renal transplant recipients. Clinical Journal of the American Society of Nephrology 6:1463-1473 DOI 10.2215/CJN.09131010. 
Sugisaki N, Wakahara T, Miyamoto N, Murata K, Kanehisa H, Kawakami Y, Fukunaga T. 2010. Influence of muscle anatomical cross-sectional area on the moment arm length of the triceps brachii muscle at the elbow joint. Journal of Biomechanics 43(14):2844-2847 DOI 10.1016/j.jbiomech.2010.06.013.

Sugisaki N, Wakahara T, Murata K, Miyamoto N, Kawakami Y, Kanehisa H, Fukunaga T. 2015. Influence of muscle hypertrophy on the moment arm of the triceps brachii muscle. Journal of Applied Biomechanics 31(2):111-116 DOI 10.1123/JAB.2014-0126.

Takarada Y, Takazawa H, Sato Y, Takebayashi S, Tanaka Y, Ishii N. 2000. Effects of resistance exercise combined with moderate vascular occlusion on muscular function in humans. Journal of Applied Physiology 88:2097-2106.

Van der Linden BJ, Koopman HF, Grootenboer HJ, Huijing PA. 1998. Modelling functional effects of muscle geometry. Journal of Electromyography and Kinesiology 8:101-109 DOI 10.1016/S1050-6411(97)00026-6.

Venturelli M, Saggin P, Muti E, Naro F, Cancellara L, Toniolo L, Tarperi C, Calabria E, Richardson RS, Reggiani C, Schena F. 2015. In vivo and in vitro evidence that intrinsic upperand lower-limb skeletal muscle function is unaffected by ageing and disuse in oldest-old humans. Acta Physiologica 215:58-71 DOI 10.1111/apha.12524.

Vikne H, Refsnes PE, Ekmark M, Medbo JI, Gundersen V, Gundersen K. 2006. Muscular performance after concentric and eccentric exercise in trained men. Medicine and Science in Sports and Exercise 38:1770-1781 DOI 10.1249/01.mss.0000229568.17284.ab.

Weijs PJ, Looijaard WG, Dekker IM, Stapel SN, Girbes AR, Oudemans-van Straaten HM, Beishuizen A. 2014. Low skeletal muscle area is a risk factor for mortality in mechanically ventilated critically ill patients. Critical Care 18:R12 DOI 10.1186/cc13189.

West DW, Burd NA, Tang JE, Moore DR, Staples AW, Holwerda AM, Baker SK, Phillips SM. 2010. Elevations in ostensibly anabolic hormones with resistance exercise enhance neither training-induced muscle hypertrophy nor strength of the elbow flexors. Journal of Applied Physiology 108(1):60-67 DOI 10.1152/japplphysiol.01147.2009.

Winwood PW, Keogh JW, Harris NK. 2012. Interrelationships between strength, anthropometrics, and strongman performance in novice strongman athletes. Journal of Strength and Conditioning Research 26:513-522 DOI 10.1519/JSC.0b013e318220db1a. 